\title{
On the size of dissociated bases
}

\author{
Vsevolod F. Lev \\ Department of Mathematics \\ University of Haifa at Oranim, Tivon 36006, Israel \\ seva@math.haifa.ac.il \\ Raphael Yuster \\ Department of Mathematics \\ University of Haifa, Haifa 31905, Israel \\ raphy@math.haifa.ac.il
}

Submitted: May 2, 2010; Accepted: May 13, 2011; Published: May 23, 2011

Mathematics Subject Classification: 05B10,11B13,05D40

\begin{abstract}
We prove that the sizes of the maximal dissociated subsets of a given finite subset of an abelian group differ by a logarithmic factor at most. On the other hand, we show that the set $\{0,1\}^{n} \subseteq \mathbb{Z}^{n}$ possesses a dissociated subset of size $\Omega(n \log n)$; since the standard basis of $\mathbb{Z}^{n}$ is a maximal dissociated subset of $\{0,1\}^{n}$ of size $n$, the result just mentioned is essentially sharp.
\end{abstract}

\section{Introduction}

Recall, that subset sums of a subset $\Lambda$ of an abelian group are group elements of the form $\sum_{b \in B} b$, where $B \subseteq \Lambda$; thus, a finite set $\Lambda$ has at most $2^{|\Lambda|}$ distinct subset sums.

A famous open conjecture of Erdős, first stated about 80 years ago (see [B96] for a relatively recent related result and brief survey), is that if all subset sums of an integer set $\Lambda \subseteq[1, n]$ are pairwise distinct, then $|\Lambda| \leq \log _{2} n+O(1)$; here $\log _{2}$ denotes the base2 logarithm. Similarly, one can investigate the largest possible size of subsets of other "natural" sets in abelian groups, possessing the property in question; say,

What is the largest possible size of a set $\Lambda \subseteq\{0,1\}^{n} \subseteq \mathbb{Z}^{n}$ with all subset sums pairwise distinct?

In modern terms, a subset of an abelian group, all of whose subset sums are pairwise distinct, is called dissociated. Such sets proved to be extremely useful due to the fact that if $\Lambda$ is a maximal dissociated subset of a given set $A$, then every element of $A$ is representable 
(generally speaking, in a non-unique way) as a linear combination of the elements of $\Lambda$ with the coefficients in $\{-1,0,1\}$. Hence, maximal dissociated subsets of a given set can be considered as its "linear bases over the set $\{-1,0,1\}$ ". This interpretation naturally makes one wonder whether, and to what extent, the size of a maximal dissociated subset of a given set is determined by this set. That is,

Is it true that all maximal dissociated subsets of a given finite set in an abelian group are of about the same size?

In this note we answer the two above-stated questions as follows.

Theorem 1 For a positive integer $n$, the set $\{0,1\}^{n}$ (consisting of those vectors in $\mathbb{Z}^{n}$ with all coordinates being equal to 0 or 1$)$ possesses a dissociated subset of size $(1+$ o(1)) $n \log _{2} n / \log _{2} 9$ (as $\left.n \rightarrow \infty\right)$.

Theorem 2 If $\Lambda$ and $M$ are maximal dissociated subsets of a finite subset $A \nsubseteq\{0\}$ of an abelian group, then

$$
\frac{|M|}{\log _{2}(2|M|+1)} \leq|\Lambda|<|M|\left(\log _{2}(2 M)+\log _{2} \log _{2}(2|M|)+2\right) .
$$

We remark that if a subset $A$ of an abelian group satisfies $A \subseteq\{0\}$, then $A$ has just one dissociated subset; namely, the empty set.

Since the set of all $n$-dimensional vectors with exactly one coordinate equal to 1 and the other $n-1$ coordinates equal to 0 is a maximal dissociated subset of the set $\{0,1\}^{n}$, comparing Theorems 1 and 2 we conclude that the latter is sharp in the sense that the logarithmic factors cannot be dropped or replaced with a slower growing function, and the former is sharp in the sense that $n \log n$ is the true order of magnitude of the size of the largest dissociated subset of the set $\{0,1\}^{n}$. At the same time, the bound of Theorem 2 is easy to improve in the special case where the underlying group has bounded exponent.

Theorem 3 Let $A$ be finite subset of an abelian group $G$ of exponent $e:=\exp (G)$. If $r$ denotes the rank of the subgroup $\langle A\rangle$, generated by $A$, then for any maximal dissociated subset $\Lambda \subseteq A$ we have

$$
r \leq|\Lambda| \leq r \log _{2} e
$$

\section{Proofs}

Proof of Theorem 1: We will show that if $n>\left(2 \log _{2} 3+o(1)\right) m / \log _{2} m$, with a suitable choice of the implicit function, then the set $\{0,1\}^{n}$ possesses an $m$-element dissociated subset. For this we prove that there exists a set $D \subseteq\{0,1\}^{m}$ with $|D|=n$ such that for every non-zero vector $s \in S:=\{-1,0,1\}^{m}$ there is an element of $D$, not orthogonal to $s$. Once this is done, we consider the $n \times m$ matrix whose rows are the elements of $D$; the columns of this matrix form then an $m$-element dissociated subset of $\{0,1\}^{n}$, as required. 
We construct $D$ by choosing at random and independently of each other $n$ vectors from the set $\{0,1\}^{m}$, with equal probability for each vector to be chosen. We will show that for every fixed non-zero vector $s \in S$, the probability that all vectors from $D$ are orthogonal to $s$ is very small, and indeed, the sum of these probabilities over all $s \in S \backslash\{0\}$ is less than 1. By the union bound, this implies that with positive probability, every vector $s \in S \backslash\{0\}$ is not orthogonal to some vector from $D$.

We say that a vector from $S$ is of type $\left(m^{+}, m^{-}\right)$if it has $m^{+}$coordinates equal to +1 , and $m^{-}$coordinates equal to -1 (so that $m-m^{+}-m^{-}$of its coordinates are equal to 0$)$. Suppose that $s$ is a non-zero vector from $S$ of type $\left(m^{+}, m^{-}\right)$. Clearly, a vector $d \in\{0,1\}^{m}$ is orthogonal to $s$ if and only if there exists $j \geq 0$ such that $d$ has exactly $j$ non-zero coordinates in the $(+1)$-locations of $s$, and exactly $j$ non-zero coordinates in the $(-1)$-locations of $s$. Hence, the probability for a randomly chosen $d \in\{0,1\}^{m}$ to be orthogonal to $s$ is

$$
\frac{1}{2^{m^{+}+m^{-}}} \sum_{j=0}^{\min \left\{m^{+}, m^{-}\right\}}\left(\begin{array}{c}
m^{+} \\
j
\end{array}\right)\left(\begin{array}{c}
m^{-} \\
j
\end{array}\right)=\frac{1}{2^{m^{+}+m^{-}}}\left(\begin{array}{c}
m^{+}+m^{-} \\
m^{+}
\end{array}\right)<\frac{1}{\sqrt{1.5\left(m^{+}+m^{-}\right)}} .
$$

It follows that the probability for all elements of our randomly chosen set $D$ to be simultaneously orthogonal to $s$ is smaller than $\left(1.5\left(m^{+}+m^{-}\right)\right)^{-n / 2}$.

Since the number of elements of $S$ of a given type $\left(m^{+}, m^{-}\right)$is $\left(\begin{array}{c}m \\ m^{+}+m^{-}\end{array}\right)\left(\begin{array}{c}m^{+}+m^{-} \\ m^{+}\end{array}\right)$, to conclude the proof it suffices to estimate the sum

$$
\sum_{1 \leq m^{+}+m^{-} \leq m}\left(\begin{array}{c}
m \\
m^{+}+m^{-}
\end{array}\right)\left(\begin{array}{c}
m^{+}+m^{-} \\
m^{+}
\end{array}\right)\left(1.5\left(m^{+}+m^{-}\right)\right)^{-n / 2}
$$

showing that its value does not exceed 1 .

To this end we rewrite this sum as

$$
\sum_{t=1}^{m}\left(\begin{array}{c}
m \\
t
\end{array}\right)(1.5 t)^{-n / 2} \sum_{m^{+}=0}^{t}\left(\begin{array}{c}
t \\
m^{+}
\end{array}\right)=\sum_{t=1}^{m}\left(\begin{array}{c}
m \\
t
\end{array}\right) 2^{t}(1.5 t)^{-n / 2}
$$

and split it into two parts, according to whether $t<T$ or $t \geq T$, where $T:=m /\left(\log _{2} m\right)^{2}$. Let $\Sigma_{1}$ denote the first part and $\Sigma_{2}$ the second part. Assuming that $m$ is large enough and

$$
n>2 \log _{2} 3 \frac{m}{\log _{2} m}(1+\varphi(m))
$$

with a function $\varphi$ sufficiently slowly decaying to 0 (where the exact meaning of "sufficiently" will be clear from the analysis of the sum $\Sigma_{2}$ below), we have

$$
\Sigma_{1} \leq\left(\begin{array}{c}
m \\
T
\end{array}\right) 2^{T} 1.5^{-n / 2}<\left(\frac{9 m}{T}\right)^{T} 1.5^{-n / 2}=\left(3 \log _{2} m\right)^{2 T} 1.5^{-n / 2},
$$

whence

$$
\log _{2} \Sigma_{1}<\frac{2 m}{\left(\log _{2} m\right)^{2}} \log _{2}\left(3 \log _{2} m\right)-\log _{2} 3 \log _{2} 1.5 \frac{m}{\log _{2} m}(1+\varphi(m))<-1,
$$


and therefore $\Sigma_{1}<1 / 2$. Furthermore,

$$
\Sigma_{2} \leq T^{-n / 2} \sum_{t=1}^{m}\left(\begin{array}{c}
m \\
t
\end{array}\right) 2^{t}<T^{-n / 2} 3^{m}
$$

implying

$$
\begin{aligned}
\log _{2} \Sigma_{2} & <m \log _{2} 3-\left(\log _{2} m-2 \log _{2} \log _{2} m\right) \log _{2} 3 \frac{m}{\log _{2} m}(1+\varphi(m)) \\
& =m \log _{2} 3\left(\frac{2 \log _{2} \log _{2} m}{\log _{2} m}(1+\varphi(m))-\varphi(m)\right) \\
& <-1
\end{aligned}
$$

Thus, $\Sigma_{2}<1 / 2$; along with the estimate $\Sigma_{1}<1 / 2$ obtained above, this completes the proof.

Proof of Theorem 2: Suppose that $\Lambda, M \subseteq A$ are maximal dissociated subsets of $A$. By maximality of $\Lambda$, every element of $A$, and consequently every element of $M$, is a linear combination of the elements of $\Lambda$ with the coefficients in $\{-1,0,1\}$. Hence, every subset sum of $M$ is a linear combination of the elements of $\Lambda$ with the coefficients in $\{-|M|,-|M|+1, \ldots,|M|\}$. Since there are $2^{|M|}$ subset sums of $M$, all distinct from each other, and $(2|M|+1)^{|\Lambda|}$ linear combinations of the elements of $\Lambda$ with the coefficients in $\{-|M|,-|M|+1, \ldots,|M|\}$, we have

$$
2^{|M|} \leq(2|M|+1)^{|\Lambda|}
$$

and the lower bound follows.

Notice, that by symmetry we have

$$
2^{|\Lambda|} \leq(2|\Lambda|+1)^{|M|}
$$

whence

$$
|\Lambda| \leq|M| \log _{2}(2|\Lambda|+1) .
$$

Observing that the upper bound is immediate if $M$ is a singleton (in which case $A \subseteq\{-g, 0, g\}$, where $g$ is the element of $M$, and therefore every maximal dissociated subset of $A$ is a singleton, too), we assume $|M| \geq 2$ below.

Since every element of $\Lambda$ is a linear combination of the elements of $M$ with the coefficients in $\{-1,0,1\}$, and since $\Lambda$ contains neither 0 , nor two elements adding up to 0 , we have $|\Lambda| \leq\left(3^{|M|}-1\right) / 2$. Consequently, $2|\Lambda|+1 \leq 3^{|M|}$, and using $(*)$ we get

$$
|\Lambda| \leq|M|^{2} \log _{2} 3 \text {. }
$$

Hence,

$$
2|\Lambda|+1<|M|^{2} \log _{2} 9+1<4|M|^{2}
$$


and substituting this back into $(*)$ we obtain

$$
|\Lambda|<2|M| \log _{2}(2|M|) .
$$

As a next iteration, we conclude that

$$
2|\Lambda|+1<5|M| \log _{2}(2|M|),
$$

and therefore, by $(*)$,

$$
|\Lambda| \leq|M|\left(\log _{2}(2|M|)+\log _{2} \log _{2}(2|M|)+\log _{2}(5 / 2)\right) .
$$

Proof of Theorem 3: The lower bound follows from the fact that $\Lambda$ generates $\langle A\rangle$, the upper bound from the fact that all $2^{|\Lambda|}$ pairwise distinct subset sums of $\Lambda$ are contained in $\langle A\rangle$, whereas $|\langle A\rangle| \leq e^{r}$.

We close our note with an open problem.

For a positive integer $n$, let $L_{n}$ denote the largest size of a dissociated subset of the set $\{0,1\}^{n} \subseteq \mathbb{Z}^{n}$. What are the limits

$$
\liminf _{n \rightarrow \infty} \frac{L_{n}}{n \log _{2} n} \text { and } \limsup _{n \rightarrow \infty} \frac{L_{n}}{n \log _{2} n} ?
$$

Notice, that by Theorems 1 and 2 we have

$$
1 / \log _{2} 9 \leq \liminf _{n \rightarrow \infty} \frac{L_{n}}{n \log _{2} n} \leq \limsup _{n \rightarrow \infty} \frac{L_{n}}{n \log _{2} n} \leq 1 .
$$

\section{References}

[B96] T. Bohman, A sum packing problem of Erdős and the Conway-Guy sequence, Proc. Amer. Math. Soc. 124 (1996), 3627-3636. 